\title{
Susceptibility Pattern of Enterococcal Isolates in a Tertiary Care Hospital against Tigecycline, Linezolid and Teicoplanin
}

\author{
Ashina Singla*, Ayushi Sharma and Ved Prakash Mamoria \\ Department of Microbiology, Mahatma Gandhi University of Medical Sciences and \\ Technology, Jaipur, India \\ *Corresponding author
}

A B S T R A C T

\section{Keywords}

Enterococcus, Susceptibility, Tigecycline, Resistance, Linezolid, Teicoplanin

\section{Article Info}

Accepted:

12 February 2019 Available Online: 10 March 2019
There has been an upsurge in the emergence of Enterococci as a nosocomial pathogen in the recent years. The emergence of vancomycin-resistant enterococci (VRE) is a cause of concern, and is very difficult to control. A study on the speciation and antimicrobial susceptibility pattern of Linezolid, Teicoplanin and Tigecycline isolated of from clinical specimens from patients in the Department of Microbiology, MGUMST was carried out in the period of January 2018 to December 2018. A total of 172 enterococcal isolates obtained from various clinical samples were included in the study. Identification and antimicrobial susceptibility testing was done on Vitek-2 Compact (bioMérieux) E. facealis (54.97\%) was the predominant enterococcal species isolated followed by E. faecium $(39.76 \%)$. The isolates were shown to have $5.84 \%$ resistance to Linezolid, $13.45 \%$ resistance to Teicoplanin and $100 \%$ sensitivity to Tigecycline. This study was performed to seek an alternative option like Linezolid, Tigecycline and Teicoplanin as vancomycin is reportedly being noted resistant in many clinical cases.

\section{Introduction}

Formerly known as Group D Streptococci, Enterococci are gram-positive bacteria that inhabit the gut of mainly immunocompromised hosts. They can grow in extreme conditions. Resistance to environmental conditions like heat ensures prolonged survival. Improper hand hygiene practices by healthcare workers results in rapid transmission of drug resistant nosocomial Enterococcal outbreaks. Most common infections caused by Enterococci are surgical wound infections, nosocomial urinary tract infections, bacteremia, septicemia and pelvic infections. Enterococcal strains have progressively acquired resistance to essentially most of the antimicrobial agents over the past few years, largely due to their resistance to many antimicrobial agents, including $\beta$-lactam antibiotics, glycopeptides, and aminoglycosides. Enterococci have become resistant to first line drugs i.e. aminoglycosides and penicillin so the alternative treatment options are Linezolid, Teicoplanin and Tigecycline $(1,2)$ 
Trimethoprim-sulfamethoxazole has also been proven to be ineffective in treating enterococcal infections. Both strains of Enterococci, E. faecalis and E. faecium are clinically resistant to Aminoglycosides. Intrinsic resistance to the $\beta$-lactam class of antibiotics is associated with the presence of a species-specific chromosomal gene, pbp5. Intrinsic resistance to cephalosporins has also been in noted in the Enterococcal species, but the molecular basis is unknown. Glycopeptide resistance is attributable to a cluster of van genes. VanA gene confers resistance to both Vancomycin and Teicoplanin. Out of the two strains, E. faecalis is globally more prevalent in clinical cases while E. faecium is intrinsically more resistant to antimicrobial agents. (3) Resistance to Vancomycin traces back to 1986 when the first case of Vancomycin resistant Enterococci was documented in Europe. Resistance in Enterococci is mediated by a cluster of Van genes. Van A and Van B genes are isolated in majority of the cases. Other genotypes are Van C, Van G and Van E. Colonization precedes infection in Vancomycin Resistant Enterococci, hence proper contact precaution and hand hygiene practices should be maintained by healthcare personnels. For alternative treatment to Vancomycin resistant Enterococci, linezolid, daptomycin, quinupristin/ dalfopristin, and tigecycline is recommended. Because of this drug resistance emerging pattern, this study was undertaken to know the effectiveness of other antibiotics like Linezolid, Teicoplanin and Tigecycline.

\section{Materials and Methods}

Enterococcal isolates obtained during a period of one year from January 2018 to December 2018 were speciated and analyzed for antibiotic susceptibility and their patterns were assessed. A total of 172 sample were collected (Table 2), out of which $86(50 \%)$ were isolated from urine, $41(23.83 \%)$ were isolated from blood, $17(9.88 \%)$ were isolated from semen, $13(7.55 \%)$ from swabs, 5 (2.9\%) from tissue and other body sites (5.84\%). Clinical specimens received in the Microbiology laboratory including Ascitic fluid, Urine, Blood, Drain, ET Secretion, Pleural fluid, Pus, Swab, Semen, Sputum and Tissue were processed as per the standard protocols. Specimens were inoculated on to solid media such as Blood agar, MacConkey agar, and also to liquid broth. All media were incubated at $37^{\circ} \mathrm{C}$. After $24 \mathrm{hrs}$ of incubation, growth was observed on solid media. The primary identification was made with basic microbiological methods using colony morphology, Gram staining and catalase test. Growth of enterococci on solid media was as follows:

On blood agar plate, enterococci produced small, round, smooth, transparent colonies and some colonies showed hemolysis alpha $(\alpha)$ or beta $(\beta)$ and some were non-hemolytic.

Gram staining was done on the colonies which morphologically resembled enterococci which showed gram positive cocci which were spherical, arranged mostly in pairs or in short chains. Catalase test was performed which was negative. Escherichia coli ATCC 25922, Staphylococcus aureus ATCC 25923, and Enterococcus faecalis ATCC 29212 were used for quality control.

The final identification and antibiotic susceptibility testing of the bacteria isolated from clinical specimens were obtained using an auto-analyzer system (bioMérieux, VITEK 2 Compact).

\section{Results and Discussion}

A total of 172 Enterococcal isolates were processed over the period of one year, out of which E. faecalis $(54.97 \%)$ was the predominant enterococcal species isolated in 
the study followed by E. faecium (39.76\%). Enterococcus avium was also isolated in 2 samples $(1.16 \%)$.

A total of 172 sample were collected, out of which $86(50 \%)$ were isolated from urine, 41 $(23.83 \%)$ were isolated from blood, 17 $(9.88 \%)$ were isolated from semen, 13 $(7.55 \%)$ from swabs, 5 (2.9\%) from tissue and other body sites $(5.84 \%)$ (Table 3$)$.

Clinical specimens received in the Microbiology laboratory including Ascitic fluid, Urine, Blood, Drain, ET Secretion, Pleural fluid, Pus, Swab, Semen, Sputum and Tissue were processed as per the standard protocols. The results of antimicrobial susceptibility are as follows:

All Enterococcal isolates were $100 \%$ susceptible to Tigecycline. Teicoplanin resistance was observed in $13.45 \%$ cases and Linezolid resistance was noted in $5.84 \%$ of the isolates (Table 1).

Statistical data of the results are as follows:

Table.1 Resistance pattern

\begin{tabular}{|l|l|}
\hline Antibiotic & Resistance \\
\hline Teicolpanin & $13.45 \%$ \\
\hline Linezolid & $5.84 \%$ \\
\hline Tigecycline & $0 \%$ \\
\hline
\end{tabular}

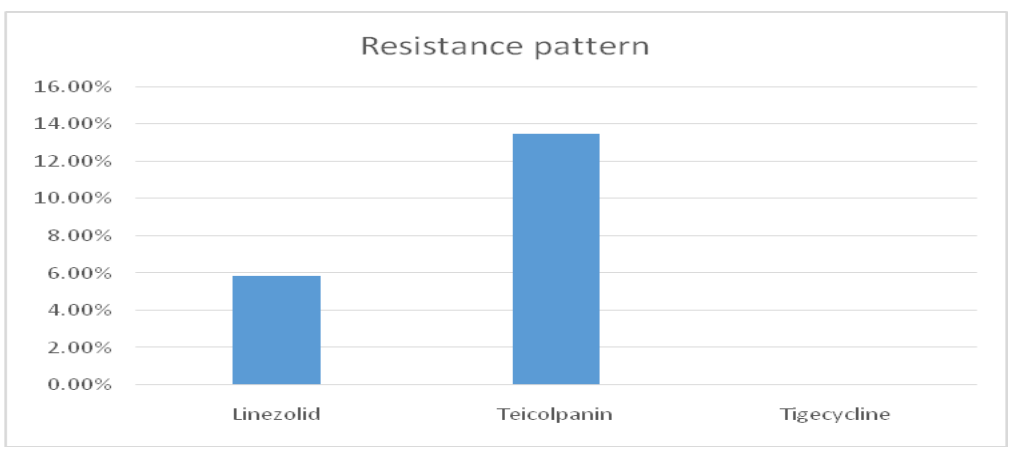

Table.2 sex wise distribution

\begin{tabular}{|c|c|}
\hline Sex & Count \\
\hline $\mathbf{F}$ & 83 \\
\hline $\mathbf{M}$ & 88 \\
\hline
\end{tabular}

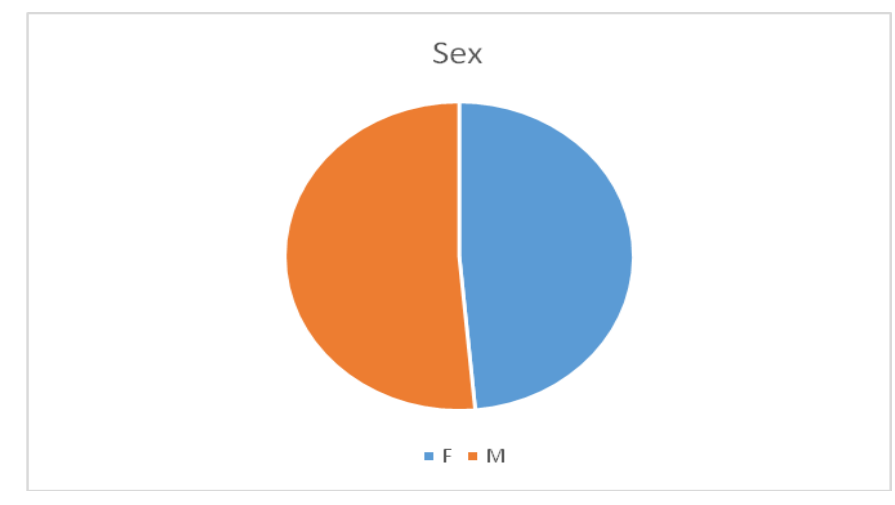


Table.3 Clinical sample wise distribution

\begin{tabular}{|c|c|}
\hline Sample & Count \\
\hline Urine & 86 \\
\hline Blood & 41 \\
\hline Swab & 13 \\
\hline Semen & 17 \\
\hline Others & 15 \\
\hline
\end{tabular}

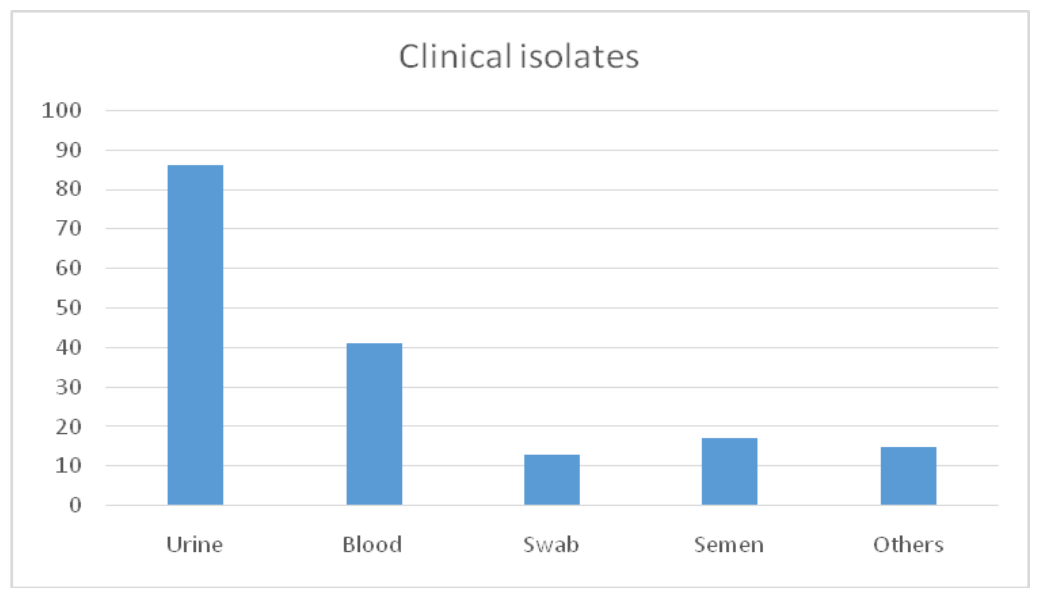

Latest studies show that Enterococcal strains are the third most isolated species in hospital acquired infections. (5) In our study the predominant species isolated was Enterococcus faecalis (54.97\%) which was followed by Enterococcus faecium (39.76\%) In our study, the prevalence of urinary tract infections caused by Enterococcal strains was $50 \%(86 / 172)$ The rationale behind may be by the transmission of the bacteria by hands of the healthcare workers while placement of the urinary catheters. (6)

Tigecycline has shown to be promising against VRE infections. (7) Tigecycline became available for clinical use in 2005. Being a broad spectrum tetracycline derivative, this novel antimicrobial agent has activity against both Gram positive and Gram negative bacteria including both aerobic and anaerobic bacteria. (8) Linezolid, belonging in the Oxazolidinone group was the first in its group to have activity against $E$. faecalis and E. faecium. It has the added advantage that it can be given orally. (9)
Our study shows that there is an emergence of resistance to Teicoplanin (13.4\%) which further reduces therapeutic options.

In conclusion, most of the enterococcal isolates from immunocomrpomised patients were resistant to Vancomycin. There was $100 \%$ sensitivity to Tigecycline, followed by Linezolid and Teicoplanin. So, the empirical therapy should be started with Linezolid or Tigecycline by clinicians

\section{References}

1. McCormick JK, Hirt H, Dunny Gm, Schlievert PM. Pathogenic mechanisms of enterococcal endocarditis. Curr Infect Dis (2000; 2(4):315-21).

2. Courvalin P. Vancomycin- resistance in gram-positive cocci. Clin Infect Dis (2006;42 suppl 1:s25-34)

3. NHSN annual update: antimicrobialresistant pathogens associated with healthcare-associated infections: annual 
summary of data reported to the National Healthcare Safety Network at the Centers for Disease Control and Prevention, 2006-2007.

4. Islam TAB, Shamsuzzaman SM. Isolation and species identification of enterococci from clinical specimen with their antimicrobial susceptibility pattern in a tertiary care hospital,

5. Jada S, Jayakumar K. Prevalence of Enterococcus species from various clinical specimens in Shrisathya Sai Medical College And Research Institute with special reference to speciation and their resistance to their resistance to vancomycin. International Journal of Medical and Clinical Research. (2012;3(4):154-160).
6. Higuita NIA, Huycke MM. Enterococcal Disease, Epidemiology, and Implications for Treatment. Infectious Diseases Section. (2014. p. 45-70)

7. 1 Cattoir V, Dowzicky MJ. A longitudinal assessment of antimicrobial susceptibility among important pathogens collected as part of the Tigecycline Evaluation and Surveillance Trial (T.E.S.T.) in France between 2004 and 2012. Antimicrob Resist Infect Control (2014; 3: 36.)

8. Rubinstein, E and Vaughan, D. Tigecycline: a novel glycylcycline. Drugs. (2005; 65: 1317-1336)

9. Vancomycin-resistant enterococci: colonization, infection, detection, and treatment. Zirakzadeh A, Patel R, Mayo Clin Proc. (2006 Apr); 81(4):529-36

\section{How to cite this article:}

Ashina Singla, Ayushi Sharma and Ved Prakash Mamoria. 2019. Susceptibility Pattern of Enterococcal Isolates in a Tertiary Care Hospital against Tigecycline, Linezolid and Teicoplanin. Int.J.Curr.Microbiol.App.Sci. 8(03): 1253-1257. doi: https://doi.org/10.20546/ijcmas.2019.803.148 